\title{
FIGO's staging classification for cancer of the ovary, fallopian tube, and peritoneum: abridged republication
}

\author{
Jaime Prat; for the FIGO Committee on Gynecologic Oncology ${ }^{1}$ \\ Department of Pathology, Hospital de la Santa Creu i Sant Pau, Autonomous University of Barcelona, Barcelona, Spain
}

Over the past quarter of a century, several scientific developments have challenged traditional concepts in ovarian cancer. First, it was recognized that ovarian cancer is not a homogeneous disease, but rather a group of diseases-each with different morphology and biological behavior. Approximately 90\% of ovarian cancers are carcinomas (malignant epithelial tumors) and, based on histopathology, immunohistochemistry, and molecular genetic analysis, at least five main types are currently distinguished: high-grade serous carcinoma (HGSC, 70\%); endometrioid carcinoma (EC,10\%); clear-cell carcinoma (CCC,10\%); mucinous carcinoma (MC, 3\%); and low-grade serous carcinoma (LGSC, <5\%) [1,2]. These tumor types (which account for $98 \%$ of ovarian carcinomas) can be reproducibly diagnosed by light microscopy and are inherently different diseases, as indicated by differences in epidemiologic and genetic risk factors; precursor lesions; patterns of spread; and molecular events during oncogenesis, response to chemotherapy, and prognosis [2,3]. Much less common are malignant germ cell tumors and potentially malignant sex cord-stromal tumors. The biomarker expression profile within a given histotype is consistent across stages. Ovarian cancers differ primarily based on histologic type.

In the era of personalized cancer medicine, reproducible

\footnotetext{
*This article is an abridged version of the staging classification published in the International Journal of Gynecology and Obstetrics in January 2014. Please cite the original article as: Prat J; FIGO Committee on Gynecologic Oncology. Staging classification for cancer of the ovary, fallopian tube, and peritoneum. Int J Gynecol Obstet 2014;124(1):1-5. Copyright Elsevier (2013)

Correspondence to Jaime Prat

Department of Pathology, Hospital de la Santa Creu I Sant Pau, Autonomous University of Barcelona, Building C, Floor 2, SantQuintí, 87-89, Barcelona 08041,Spain.E-mail:jprat@santpau.cat
}

histopathologic diagnosis of tumor cell type is a sine qua non for successful treatment. Different tumor histotypes respond differently to chemotherapy. The International Federation of Gynecology and Obstetrics (FIGO) Committee on Gynecologic Oncology unanimously agreed that histologic type should be designated at staging.

The finding of high-grade serous tubal intraepithelial carcinoma (STIC), in patients with BRCA mutation undergoing risk-reducing salpingo-oophorectomy (RRSO)[4] also influenced the new FIGO staging. Although STIC is capable of metastasizing and, therefore, cannot be considered a true carcinoma in situ, compelling evidence for a tubal origin of BRCA-positive HGSC has accumulated over the past decade $[5,6]$. The relative proportion of HGSCs of ovarian and tubal derivation is unknown, mainly because tumor growth in advanced-stage cancers conceals the primary site. Even in cases involving BRCA mutation, evidence of a tubal origin of HGSCs is incomplete and a multicentric origin of these tumors cannot be excluded.

The process of the proposed changes to the staging of ovarian, fallopian tube, and primary peritoneal cancer started three years ago under the leadership of the Chair of the FIGO Committee on Gynecologic Oncology, Professor Lynette Denny. The proposal was sent to all relevant gynecologic oncology organizations and societies worldwide. The new staging was reached by consensus of those participating in the FIGO meeting held in Rome, Italy, on October 7, 2012 and approved two weeks later.

The following is the consensus agreement that resulted from these efforts and represents new criteria for staging of these gynecologic cancer (Table 1). 
Table 1. 2014 FIGO ovarian, fallopian tube, and peritoneal cancer staging system and corresponding TNM

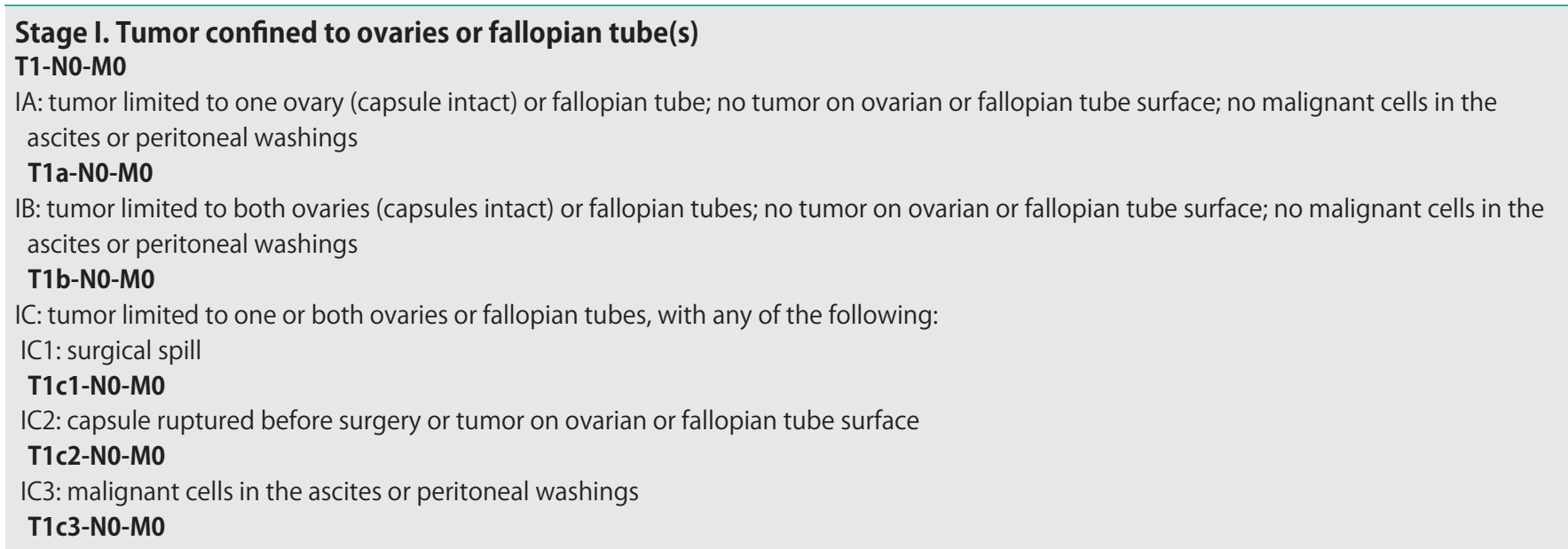

Stage II. Tumor involves one or both ovaries or fallopian tubes with pelvic extension (below pelvic brim) or primary peritoneal cancer T2-NO-MO

IIA: extension and/or implants on uterus and/or fallopian tubes and/or ovaries

T2a-NO-MO

IIB: extension to other pelvic intraperitoneal tissues

T2b-NO-MO

Stage III. Tumor involves one or both ovaries or fallopian tubes, or primary peritoneal cancer, with cytologically or histologically confirmed spread to the peritoneum outside the pelvis and/or metastasis to the retroperitoneal lymph nodes

T1/T2-N1-M0

IIIA1: positive retroperitoneal lymph nodes only (cytologically or histologically proven):

IIIA1(i) Metastasis up to $10 \mathrm{~mm}$ in greatest dimension

IIIA1(ii) Metastasis more than $10 \mathrm{~mm}$ in greatest dimension

IIIA2: microscopic extrapelvic (above the pelvic brim) peritoneal involvement with or without positive retroperitoneal lymph nodes

T3a2-N0/N1-M0

IIIB: macroscopic peritoneal metastasis beyond the pelvis up to $2 \mathrm{~cm}$ in greatest dimension, with or without metastasis to the retroperitoneal lymph nodes

T3b-N0/N1-M0

IIIC: macroscopic peritoneal metastasis beyond the pelvis more than $2 \mathrm{~cm}$ in greatest dimension, with or without metastasis to the retroperitoneal lymph nodes (includes extension of tumor to capsule of liver and spleen without parenchymal involvement of either organ) T3c-N0/N1-M0

\section{Stage IV. Distant metastasis excluding peritoneal metastases}

Stage IVA: pleural effusion with positive cytology

Stage IVB: parenchymal metastases and metastases to extra-abdominal organs (including inguinal lymph nodes and lymph nodes outside of the abdominal cavity)

Any T, any N, M1

\section{MAIN CHANGES}

The primary site (i.e., ovary, fallopian tube, or peritoneum) should be designated where possible. In some cases, it might not be possible to delineate the primary site clearly; such cases should be listed as "undesignated". The histologic type should be recorded.

Stage I ovarian or fallopian tube cancer is confined to the ovaries or the fallopian tubes and peritoneal fluid/washings.
Tumor rupture, surface involvement by tumor cells or presence of malignant cells in the ascites or peritoneal washings warrants a stage of IC. It is not possible to have stage I peritoneal cancer.

Stage II ovarian cancer comprises a small and heterogeneous group making up less than $10 \%$ of ovarian cancers. It is defined as extension or metastasis to extraovarian/ extratubal pelvic organs and may include curable tumors that have directly extended to adjacent organs but have 
not yet metastasized, as well as tumors that have seeded the pelvic peritoneum by metastasis and, therefore, have a poor prognosis. The Committee felt that subdividing this small category further into IIB1 and IIB2 (i.e., microscopic and macroscopic pelvic peritoneal metastases) was not based on evidence/biology. All stage II disease is treated with adjuvant chemotherapy, so subclassification is not essential. Also, the old substage IIC (i.e., IIA or IIB but with tumor on surface, capsule ruptured, or ascites or positive peritoneal washing) was considered redundant and eliminated.

Most ovarian cancers are HGSCs that usually present in stage III, with the vast majority (84\%) stage IIIC [7]. These tumors characteristically spread along peritoneal surfaces involving both pelvic and abdominal peritoneum. Less than $10 \%$ of ovarian carcinomas extend beyond the pelvis with exclusively retroperitoneal lymph node involvement. Evidence in the literature indicates that these cases have a better prognosis than that of tumors with abdominal peritoneal involvement [8-14]. The new staging includes a revision of stage III patients and assignment to stage IIIA1 based on spread to the retroperitoneal lymph nodes without intraperitoneal dissemination. Stage IIIA1 is further subdivided into IIIA1(i) (metastasis $\leq 10 \mathrm{~mm}$ in greatest dimension) and IIIA1(ii) (metastasis $>10$ $\mathrm{mm}$ in greatest dimension), even if there are no retrospective data supporting quantification of the size of metastasis in IIIA1. Involvement of retroperitoneal lymph nodes must be proven cytologically or histologically.

Stage IV is defined as distant metastasis and includes patients with parenchymal liver/splenic metastases and extra-abdominal metastases; $12 \%$ to $21 \%$ of patients present with stage IV disease [7]. Extension of tumor from omentum to spleen or liver (stage IIIC) should be differentiated from isolated parenchymal metastases (stage IVB).

\section{ACKNOWLEDGMENTS}

${ }^{1}$ Committee members: H. Belhadj (Switzerland), J. Berek (USA), A. Bermudez (Argentina), N. Bhatla (India), J. Cain (USA), L. Denny (Chair; South Africa), K. Fujiwara (Japan), N. Hacker (Australia), E. Åvall-Lundqvist (Sweden), D. Mutch (USA), F. Odicino (Italy), S. Pecorelli (Italy), J. Prat (Spain), M. Quinn (Co-chair; Australia), M.A-F. Seoud (Lebanon), S.K. Shrivastava (India).

\section{REFERENCES}

1. Kurman RJ, Carcangiu ML, Herrington CS, Young RH. WHO classification of tumours of female reproductive organs. 4th ed. Lyon: International Agency for Research on Cancer; 2014.

2. Prat J. Ovarian carcinomas: five distinct diseases with different origins, genetic alterations, and clinicopathological features. Virchows Arch 2012;460:237-49.

3. Gilks CB, Prat J. Ovarian carcinoma pathology and genetics: recent advances. Hum Pathol 2009;40:1213-23.

4. Piek JM, van Diest PJ, Zweemer RP, Jansen JW, Poort-Keesom RJ, Menko FH, et al. Dysplastic changes in prophylactically removed Fallopian tubes of women predisposed to developing ovarian cancer. J Pathol 2001;195:451-6.

5. Callahan MJ, Crum CP, Medeiros F, Kindelberger DW, Elvin JA, Garber $J E$, et al. Primary fallopian tube malignancies in BRCA-positive women undergoing surgery for ovarian cancer risk reduction. J Clin Oncol 2007;25:3985-90.

6. Kindelberger DW, Lee Y, Miron A, Hirsch MS, Feltmate C, Medeiros $F$, et al. Intraepithelial carcinoma of the fimbria and pelvic serous carcinoma: evidence for a causal relationship. Am J Surg Pathol 2007;31:161-9.

7. Heintz AP, Odicino F, Maisonneuve P, Quinn MA, Benedet $J$, Creasman WT, et al. Carcinoma of the ovary. FIGO 26th Annual Report on the Results of Treatment in Gynecological Cancer. Int J Gynaecol Obstet 2006;95 Suppl 1:S161-92.

8. Onda T, Yoshikawa H, Yasugi T, Mishima M, Nakagawa S, Yamada $M$, et al. Patients with ovarian carcinoma upstaged to stage III after systematic lymphadenctomy have similar survival to Stage I/ I| patients and superior survival to other Stage III patients. Cancer 1998;83:1555-60.

9. Kanazawa K, Suzuki T, Tokashiki M. The validity and significance of substage IIIC by node involvement in epithelial ovarian cancer: impact of nodal metastasis on patient survival. Gynecol Oncol 1999;73:237-41.

10. Panici PB, Maggioni A, Hacker N, Landoni F, Ackermann S, Campagnutta $\mathrm{E}$, et al. Systematic aortic and pelvic lymphadenectomy versus resection of bulky nodes only in optimally debulked advanced ovarian cancer: a randomized clinical trial. J Natl Cancer Inst 2005;97:560-6.

11. Cliby WA, Aletti GD, Wilson TO, Podratz KC. Is it justified to classify patients to Stage IIIC epithelial ovarian cancer based on nodal involvement only? Gynecol Oncol 2006;103:797-801.

12. Ferrandina G, Scambia G, Legge F, Petrillo M, Salutari V. Ovarian cancer patients with "node-positive-only" Stage IIIC disease have a more favorable outcome than Stage IIIA/B. Gynecol Oncol 2007;107:154-6.

13. Baek SJ, Park JY, Kim DY, Kim JH, Kim YM, Kim YT, et al. Stage IIIC epithelial ovarian cancer classified solely by lymph node metastasis has a more favorable prognosis than other types of stage IIIC epithelial ovarian cancer. J Gynecol Oncol 2008;19:223-8.

14. Bakkar R, Gershenson D, Fox P, Vu K, Zenali M, Silva E. Stage IIIC ovarian/peritoneal serous carcinoma: a heterogeneous group of patients with different prognoses. Int J Gynecol Pathol 2014;33:302-8. 